\title{
A modelling methodology for natural dam-river network systems
}

\author{
X. Zhuan ${ }^{\mathrm{a}, \mathrm{b}}, \mathrm{G}$. Zheng $^{\mathrm{b}}, \mathrm{X} . \mathrm{Xia}^{\mathrm{a}, \mathrm{b}, *}$ \\ a Department of Electrical, Electronic and Computer Engineering, University of Pretoria, Pretoria 0002, South Africa \\ ${ }^{\mathrm{b}}$ Department of Automation, Wuhan University, Wuhan 430072, China
}

\section{A R T I C L E I N F O}

\section{Article history:}

Received 2 October 2007

Accepted 7 October 2008

\section{Keywords:}

Open channel

Natural river network

Modelling

System identification

\begin{abstract}
A B S T R A C T
An approach for modelling a natural dam-river network system is proposed in this paper. Generally, the relationships among the variables of a natural dam-river network system are complex and difficult to describe. In this paper, some simple relationships among the water levels measured at a limited number of points of the network system are presented in such a way that a simple model is achieved. The model is identified and validated with the real time operational data. An example is given and the result shows the feasibility of the modelling methodology. It is believed that the proposed approach can be used in the operation of natural dam-river or river network systems.
\end{abstract}

(c) 2008 Elsevier Ltd. All rights reserved.

\section{Introduction}

Owing to the scarcity of water resources, modelling and control of water resources in open channel flows have attracted a lot of research in recent years. The common natural examples of open channels are water flows in rivers and streams, while manmade examples are irrigation canals and sewer lines. Generally, the channel bed and channel geomorphological features of a manmade channel are regular and the material types of the channel are uniform, while the channel bed and channel geomorphological features of a natural channel are irregular and the material types of the channel vary in different locations.

Except for flood control, the constant subject of study is mainly the dynamics of manmade channels. This is because the dynamics are regular and the dynamical parameters are regular in manmade channels, enabling a model-based study. A recent survey of models can be seen in Zhuan and Xia (2007), where the models are classified into physical principle models and data-driven models. Two principles are used in water flow dynamics. One is the so-called Saint Venant equations (Chow, 1959). From Saint Venant equations, a class of models are derived (discretized in Balogun, Hubbard, \& De Vries, 1988; Garcia, Hubbard, \& De Vries, 1992; Georges, 1994, and linearized in Baume \& Sau, 1997; Chentouf, Xu, \& Boulbrachene, 2001; Duviella, Charbonnaud, Chiron, \& Carrillo, 2005; Litrico, 2001; Litrico \& Georges, 1997, 1999a, 1999b, 2001; Litrico, Georges, \& Trouvat, 1998). The other principle is the water volume or mass balance principle (Corriga,

\footnotetext{
* Corresponding author at: Department of Electrical, Electronic and Computer Engineering, University of Pretoria, Pretoria 0002, South Africa. Tel.: +27 12 4202165; fax: +27123625000.

E-mail addresses: xtzhuan@whu.edu.cn (X. Zhuan), glzheng@whu.edu.cn (G. Zheng), xxia@postino.up.ac.za (X.Xia).
}

Patta, Sanna, \& Usai, 1979; Corriga, Sanna, \& Usai, 1983; Schuurmans, Bosgra, \& Brouwer, 1995; Schuurmans, Hof, Dijkstra, Bosgra, \& Brouwer, 1999), with which some volume (mass) balance models are presented. The parameters in data-driven models are identified from real time data. Such models include black-box models in Elfawal-Mansour, Georges, and Ohnishi (2000), greybox models in Weyer (2001, 2003a, 2003b), Ooi and Weyer (2001), Ooi, Weyer, and Campi (2003), Ooi, Krutzen, and Weyer (2005), Li, Cantoni, and Weyer (2005), Mareels et al. (2005) and Maxwell and Warnick (2006), high order transfer function models in Sawadogo, Faye, Malaterre, and Mora-Camino (1998) and Sawadogo, Faye, Benhammou, and Akouz (2000) and neural network models in Toudeft and Gallinari $(1996,1997)$. Neural network techniques are also employed in Paravan, Stokelj, and Golob (2004) to forecast theshort-term water inflow in a hydroelectrical power plant. The difference between the black-box models and the grey-box models is that the grey-box models partially satisfy the volume or mass balance principle.

For natural channels, although the dynamics can be described by Saint Venant equations, the equations are not as useful as in manmade channels because the parameters in Saint Venant equations vary with respect to the different space and time coordinates. The variation of the parameters leads to difficulties in studying the flow dynamics and, therefore, the control strategies of the flow dynamics with a mathematical model. The control objective of open channels is to transport the water resources from one area to (an)other area(s) such that water flows and water levels meet the demands of users, the ecology, safety requirements (flood control), navigation control, pollution control and decreasing water waste.

For a natural dam-river network system, the existing control strategies are scheduled on the basis of experimental operation and information in a limited local area (points) for a limited 
period without accurate prediction. For example, in a floodpreventing hydraulic structure-Enclosing Dike-a dike surrounding an area with gates/pumping stations connecting the outside and inside of the dike, is operated in a very simple way. When the water level at a point inside the area is detected to be higher than the security water level, the water in the inside area will immediately be pumped out through the pumping station. When the water level is so low that the environment of the inside area has too little water, the gate will immediately be opened to introduce water from outside. All those operations are based on accumulated experience and on the observation of present local states without considering the whole system and predicting the development trends. This kind of decision-making concerning control strategies cannot realize global optimal control for a large and complicated river network system. The study subject in this paper is such a kind of large and complicated natural river network system. An investigation will be conducted to obtain a mathematical model for such a network system and it is expected that the model will be able to facilitate the design of control strategies.

A kind of methodology for modelling a natural dam-river network system with simple measurements and variables is proposed in this paper. Such a dam-river system is very complex and at present no simple mathematical model is being applied to the system operation. A model established in line with the proposed approach is very similar to the grey-box model in Weyer (2001, 2003a, 2003b), Ooi and Weyer (2001), Ooi et al. (2003, 2005), Li et al. (2005), Mareels et al. (2005), and Maxwell and Warnick (2006), but is much simpler because it includes water level measurements at some points and control variables, while in the references the flow rates are included in the models. As is known, the flow rates are difficult to measure, especially for natural channels (the velocity distribution in a cross-section of the channel is very complex). So the model in this paper is much simpler. The model structure is proposed based on the simplification of some hydraulic principles. The parameters in the model can be identified with experimental or real time operational data. For the parameter identification, various methods could be applied. In this paper, the model identification and validation methods are not detailed. To show the potential application of the proposed approach, an example of an Enclosing Dike system is given.

The contribution of this paper lies in two aspects. The first is that, different from existing papers, the modelling methodology for a natural river network system is studied in this paper. The second is that in the proposed "grey-box" model, only water level and some control variables are included, which avoids the requirement of measuring the flow rates (difficult to obtain in a natural channel).

The structure of this paper is: the modelling methodology is proposed in Section 2, while in Section 3 an application example is given to show the feasibility of the modelling methodology. The conclusions are in Section 4.

\section{Modelling methodology}

The variables in modelling a dam-river network considered in this paper are only the water levels at different points. For simplicity, the control variables in this case are the gate openings. The relationship between the water levels can be classified into three types, as shown in Fig. 1: gate connection, channel connection and non-channel connection. Gate connection indicates that there is one and only one gate structure between the two points with water levels (upstream and downstream of a gate) measured. Channel connection means there is a visible

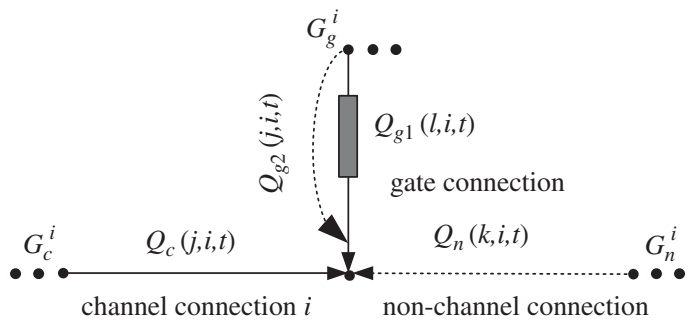

Fig. 1. Connection types.

channel with water flow connecting the two points with water levels measured. When the connection between two points does not belong to a gate connection or channel connection, it is called a non-channel connection.

In this paper, the flow rates in channels are assumed not to be measured, which is practical, for the flow rates are difficult to measure (flow rates are not measured practically). However, it is assumed that the flow rates vary in a small range, at least over a short time period.

The flow through a porous medium (aquifer) in a non-channel connection is generally expressed by Darcy's Law formulated on the basis of experiments (refer to Todd \& Mays, 2005). The calculation of flow through a porous medium is usually in relation to flow nets, which is very complex. According to Darcy's Law, the flow rate through a porous medium is proportional to the head loss (generally the difference between water levels) and hydraulic conductivity or intrinsic permeability, and inversely proportional to the length of the flow path (distance). In this paper, such a flow rate $Q_{n}(i, j, t)$ to point $j$ from point $i$ is just approximated by

$Q_{n}(i, j, t)=\alpha_{n}(i, j) y\left(i, t-t_{d}(i, j)\right)+\beta_{n}(i, j) y(j, t)$,

where $y\left(i, t-t_{d}(i, j)\right)$ is the water level at point $i$ at the time $t-t_{d}(i, j), y(j, t)$ is the water level at point $j$ at the time $t, t_{d}(i, j)$ is the time delay and $\alpha_{n}(i, j)$ and $\beta_{n}(i, j)$ are coefficients. This equation can also be approximated by a higher order series, but it is simply approximated by a first order function in this paper.

For gate connection, the relationship between the water levels inside and outside the gate depends on two items. The first is the flow through the gate when the gate is open. The second is the flow through a porous medium.

When the gate is open, the flow through the gate depends on the water levels on both sides of the gate, the gate opening and the gate type. In Eurén and Weyer (2007), the flow rate $Q_{g}(i, j, t)$ for an undershot gate is approximated by

$Q_{g 1}(i, j, t)=c \cdot \delta d_{g}(t) \cdot \operatorname{sgn}(y(i, t)-y(j, t)) \sqrt{|y(i, t)-y(j, t)|}$,

where $c$ is a constant determined by the gate characteristics, $\delta d_{g}(t)$ is the gate opening and $y(i, t)$ and $y(j, t)$ are the water levels at points $i$ and $j$ (the water levels on both sides of the gate). The signum function of a real number $x$ is defined as follows:

$\operatorname{sgn} x= \begin{cases}-1 & \text { if } x<0 \\ 0 & \text { if } x=0 \\ 1 & \text { if } x>0\end{cases}$

The flow rate through a porous medium in a gate connection is in the same form as Eq. (1), i.e.,

$Q_{g 2}(i, j, t)=\alpha_{g}(i, j) y\left(i, t-t_{d}(i, j)\right)+\beta_{g}(i, j) y(j, t)$.

So for a gate connection, the flow rate is described by

$Q_{g}(i, j, t)=Q_{g 1}(i, j, t)+Q_{g 2}(i, j, t)$.

The flow rate of a channel connection can be calculated by Saint Venant equations, a group of partial differential equations. According to Darcy's Law, with the assumption that the flow 
speed varies slowly, the flow rate $Q_{c}(i, j, t)$ to point $j$ at time $t$ in the channel connecting to point $i$ is approximated by

$Q_{c}(i, j, t)=\alpha_{c}(i, j) y\left(i, t-t_{d}(i, j)\right)+\beta_{c}(i, j) y(j, t)$

with $y(i, t)$ and $y(j, t)$ water levels at points $i, j$ at time $t, t_{d}(i, j)$ time delay and $\alpha_{c}(i, j)$ and $\beta_{c}(i, j)$ coefficients.

Another assumption in this paper is that the water volume storage in the neighbourhood of a point $i$ is proportional to the water level $y(i, t)$. With this assumption, according to the mass balance principle, the water level $y(i, t)$ could be predicted as follows:

$$
\begin{aligned}
\gamma(i) \frac{\mathrm{d} y(i, t)}{\mathrm{d} t}= & \sum_{l \in G_{g}^{i}} Q_{g 1}(l, i, t)+\sum_{l \in G_{g}^{i}} Q_{\mathrm{g} 2}(l, i, t) \\
& +\sum_{j \in G_{c}^{i}} Q_{c}(j, i, t)+\sum_{k \in G_{n}^{i}} Q_{n}(k, i, t)+e(t),
\end{aligned}
$$

where $G_{c}^{i}, G_{n}^{i}$ and $G_{g}^{i}$ are the sets in which the elements with respect to point $i$, respectively, belong to a channel connection, a non-channel connection and a gate connection, $\gamma(i)$ is a coefficient and $e(t)$ is the disturbance or unmodelled flow, such as rainfall and irrigation flow.

Comparing Eqs. (1), (4) and (6) reveals that they are in the same form. Let $G^{i}=G_{g}^{i} \cup G_{c}^{i} \cup G_{n}^{i}$. With the above equations combined, the following holds:

$$
\begin{aligned}
\frac{\mathrm{d} y(i, t)}{\mathrm{d} t}= & \sum_{k \in G^{i}}\left(\alpha(k, i) y\left(k, t-t_{d}(k, i)\right)+\beta(k, i) y(i, t)\right) \\
& +\sum_{l \in G_{g}^{i}} v(i) Q_{g_{1}}(l, i, t)+e(t)
\end{aligned}
$$

where $\alpha, \beta$, and $v$ are coefficients and can be identified with real data.

Model (8) can be discretized with various discretization methods, resulting in different kinds of discrete models. In this paper, for simplicity, an autoregressive model is chosen with exogenous input (ARX model) in the form

$$
\begin{aligned}
A\left(q^{-1}\right) y(K) & =B\left(q^{-1}\right) u(K)+P\left(q^{-1}\right) w(K)+Q\left(q^{-1}\right) e(K) \\
& =\left[B\left(q^{-1}\right) P\left(q^{-1}\right)\right][u(K) w(K)]^{\mathrm{T}}+e_{s}(K)
\end{aligned}
$$

with $K$ the discrete time, $e(K)$ unknown disturbance, $y(K)=y(i, K)$ $i$ th output, $u(K)=Q_{\mathrm{g} 1}(K)$ control input, $w(K)=(y(k, K))_{k \in G^{i}}$ uncontrollable input, $e_{s}(K)=Q / \operatorname{Ae}(K)$ the unknown disturbance in a new form, $q^{-1}$ delay operator and $t$ discrete sampling time series. From this equation, it can be noticed that the effect of $e_{s}(K)$ depends on the filter $Q / A$ and the characteristics of $e(K)$.

To date, there is no accurate model to describe the dynamics of a natural dam-river network system. Even the introduction of Saint Venant equations is based on some assumptions and furthermore, the parameters in Saint Venant equations are variable in natural channels and difficult to determine. Model (9) is based on some practical assumptions and the coefficients are determined by system identification with realtime data. As for the slow-varying coefficients, they can be updated by identification with the most recent operational data. Model (9) is very simple compared with Saint Venant equations or Darcy's Law. Only the water levels appear in model (9).

It should be noticed that various model identification approaches could be employed with the proposed modelling methodology.

The more measurement points there are, the more accurate the model is. However, when the number of measurement points is limited, the measurement points can be scheduled at the places where there are obvious geomorphological variations to improve the accuracy of model (9), i.e., the geomorphological features can be considered in scheduling the measurement points to improve the model accuracy. For example, a measurement point can be scheduled at the place where the cross-section changes abruptly, to improve the accuracy of the model.

Another remark is that (9) is obtained from the simple model (8) by employing a discretization scheme. This discretization scheme could be, for instance, analogous to the finite element scheme in solving the Saint Venant equations. This may result in high orders in terms of $q^{-1}$ in model (9), therefore with higher accuracy than (8).

\section{Application of modelling methodology}

An example is given in this section to show the feasibility of the proposed modelling methodology. The example, namely Enclosing Dike, is a typical kind of hydraulic structure, composed of an Enclosing Dike, sluice gates and pumping stations, which is constructed beside a river connected to the sea in South China to dampen the influence of the large tidal variations and to allow the consolidation of upstream urban infrastructure.

Sixiang Enclosing Dike, as shown in Fig. 2, located in Nanhai city, Guangdong Province, protects the city from flooding and ensures the environmental balance. It is composed of 17 sluice gates, ten pumping stations and a long Enclosing Dike. The main aim is to drain away flooded water when the water level in the Enclosing Dike exceeds the security level, and to draw water from the river when the Enclosing Dike is in need of water. In the past, the sluice gates and pumping stations were operated by hand according to the experience of the operators. Equipped with the automatic operation facilities and optical fibre cabling network built in recent years, all information is collected and transmitted to a supervising and controlling centre. The information includes the running state of the facilities, such as gate openings, the running state of the pumps, and water levels at some points inside and outside the Enclosing Dike, and even video images of the supervised points. Thus the operators can know the situations of all pumping stations and sluice gates, and furthermore make decisions and send operational commands to the stations and gates. With the project applied, the level of Enclosing Dike automatic control is advanced and reliability is enhanced. It is possible to realize optimal control in the global viewpoint of Enclosing Dike. At present control is still local and the level of performance depends on the experience of the operators. The difficulty in the application of global automatic control lies in the absence of a model describing the whole dynamics of an Enclosing Dike.

In the supervising and controlling centre, data are collected from pumping stations and undershot sluice gates (hydraulic

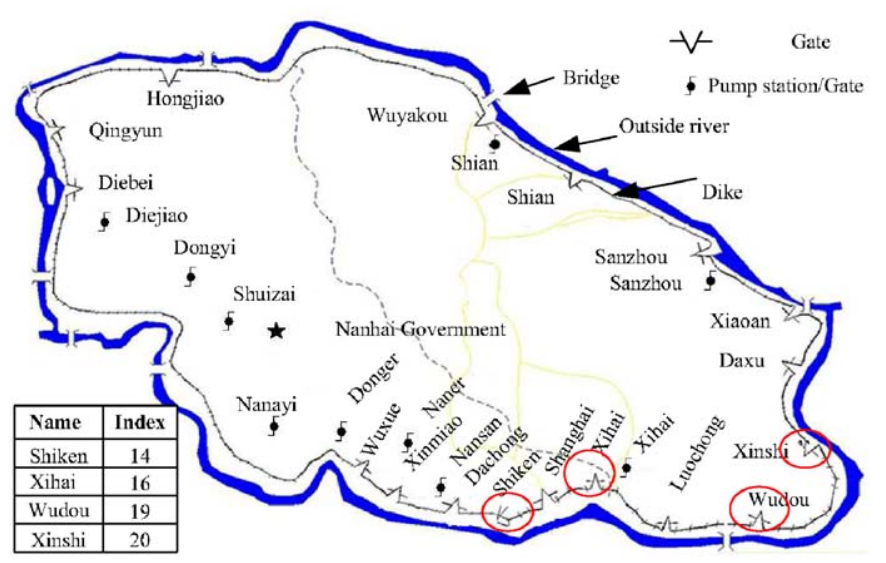

Fig. 2. Sixiang Enclosing Dike. 
structures), including water levels inside and outside the hydraulic structures, the pump status (on/off) and gate openings. At present, gate openings only have two states: open and closed, where open state means that the plate bottom is over the water surface and closed state means there is no water flowing through the gate.

The measured variables in the above-mentioned project are water levels $y_{n j}(i)$ and $y_{w j}(i)$ inside and outside the hydraulic structures ( $i$ is the index of the hydraulic structure). The control variables are also the states of the sluice gates and pumps, i.e., the gate openings $\delta d_{g}(i)$ and pump state $\delta d_{p}(i)$.

The available operational data of the Enclosing Dike were limited to four sluice gates (the indexes of the hydraulic structures are $14,16,19$ and 20) and one pumping station (its index is 17 , it is connected to structure 16 and the two structures are almost in the same place). The data for the other hydraulic structures were not continuously measured or recorded. During the period of data sampling, there was no record of the operation of the pumps. Because the project is used to show the applicability of the modelling approach in Section 2, the above-mentioned data with only four points in the Enclosing Dike are enough.

The data have the following features:

- The period over which the data were collected is about 17 days.

- The measured water levels are the Yellow Sea's altitudes.

- The data were collected with asynchronous samples.

- The time intervals of the data are about $5 \mathrm{~min}$.

- Some data were missed in the record.

- Taking into consideration the installation of the sensors and calculation formula, there are different offsets for various water levels.

Based on the available data, the model as in (10) is expected to be established for the Enclosing Dike. In (10), $Q_{g 1}$ are in the form of (2), and the coefficients $A(i), B(i)$ and $P(i)$ will be identified with the operational data.

$A\left(i, q^{-1}\right) y(i, K)=B\left(i, q^{-1}\right) u(i, K)+P\left(i, q^{-1}\right) w(i, K)+e_{S_{i}}(K)$,

$i=14,16,19,20$,

where $y(i, K)=y_{n j}(i, K), u(i, t)=Q_{g 1}(i, t)$,

$w(14, K)=\left[y_{w j}(14, K), y_{n j}(16, K), y_{n j}(19, K), y_{n j}(20, K)\right]^{\mathrm{T}}$,

$w(16, K)=\left[y_{w j}(16, K), y_{n j}(14, K), y_{n j}(19, K), y_{n j}(20, K)\right]^{\mathrm{T}}$,

$w(19, K)=\left[y_{w j}(19, K), y_{n j}(14, K), y_{n j}(16, K), y_{n j}(20, K)\right]^{\mathrm{T}}$,

$w(20, K)=\left[y_{w j}(20, K), y_{n j}(14, K), y_{n j}(16, K), y_{n j}(19, K)\right]^{\mathrm{T}}$.

The water levels outside the dike are mainly influenced by the tidal process and flow rate from upstream in the river. These can be estimated by using the approach proposed in this paper in a wider system incorporating the tidal prediction. In this paper, the water levels outside the dike are assumed to be known.

In the above model, $Q_{g 1}$ is thought of as the control variable. In modelling the Enclosing Dike, pump flow rates of pumping stations can also be expressed in the form of control variables and thus the control problem of the Enclosing Dike could be studied.

\subsection{Data preprocessing}

The sampled data were processed prior to being used for identification. The preprocess includes virtual synchronization, offset correction and missing data estimation. To illustrate the efficiency of the modelling approach, a common algorithm for model identification is employed in this study for model parameter identification.
The sampled data were not synchronous because they were independently collected at different points and it was inconvenient to synchronize the sampling time for them. However, in model (10), the data are required to be synchronous. So, a kind of virtual synchronization is employed to preprocess the original data. The time series for model (10) is predetermined as $t_{0}, \ldots, t_{0}+K T, \ldots, t_{0}+N T$ with $t_{0}$ the referenced time, $N$ the time length and $K=1, \ldots, N-1$. The data are virtually synchronized by way of linear interpolation.

The offsets in the data for different variables differ. In the presented model, it is difficult to know the offsets of each measurement. The measurement sensors were installed individually and calibrated with respect to the Yellow Sea's altitude according to the workers' experience. Each measurement has a different offset. To eliminate this kind of system error, it is better to employ a second-order model for the model structure to decrease the effect of offsets.

The missing data were corrected by linear interpolation. If the time interval of the continuous missing data is short, such a method will not lead to a large error in the model. Otherwise, the interpolation may result in a large error. In this paper, it is assumed that there are few time intervals with data missing. In fact, unless something wrong happens with the communication network, there are few data missing.

\subsection{Model identification and validation}

The experimental data were collected over a period of about 17 days $(4700 \times 5 \mathrm{~min})$, in which half of the length (2500 sampling points) were used for the model identification, and the remaining data for the model validation.

The model structure is a second order ARX model $\left(A\left(q^{-1}\right)\right.$, $B\left(q^{-1}\right)$ and $P\left(q^{-1}\right)$ are polynomials of $q^{-1}$ of two consecutive orders). The disturbance is not considered for it is difficult to know its distribution class. A second order ARX model is employed and expected to decrease the influence of the offsets of measurements and the disturbance in a limited period.

The next step is to identify the time delays in (10). The delays in the variables, gate flows and water levels outside the gate are 1. The other time delays are estimated based on comparison of ARX models with different delays in the range of $[1,100]$. The result is shown in Table 1.

The parameters in an ARX model (9) are estimated with the least squares method, and the results are shown as follows.

If $y(t)$ is the water level $y_{n j}(14, t)$, then the parameters are

$A\left(14, q^{-1}\right)=1-1.456 q^{-1}+0.459 q^{-2}$,

$B\left(14, q^{-1}\right)=0.073 q^{-1}-0.014 q^{-2}$,

$P\left(14, q^{-1}\right)=\left[\begin{array}{c}-0.004 q^{-1}+0.010 q^{-2} \\ -0.039 q^{-14}+0.041 q^{-15} \\ -0.085 q^{-67}+0.085 q^{-68} \\ -0.007 q^{-97}+0.009 q^{-98}\end{array}\right]$.

Table 1

Time delays identified from the data.

\begin{tabular}{lllll}
\hline Output & \multicolumn{2}{l}{ Input } & & \\
\cline { 2 - 5 } & 14 & 16 & 19 & 20 \\
\hline 14 & - & 14 & 67 & 97 \\
16 & 72 & - & 19 & 38 \\
19 & 98 & 35 & - & 8 \\
20 & 95 & 32 & 14 & - \\
\hline
\end{tabular}


If $y(t)$ is the water level $y_{n j}(16, t)$, then the parameters are

$A\left(16, q^{-1}\right)=1-1.342 q^{-1}+0.346 q^{-2}$,

$B\left(16, q^{-1}\right)=0.256 q^{-1}-0.061 q^{-2}$,

$P\left(16, q^{-1}\right)=\left[\begin{array}{c}-0.039 q^{-1}+0.0484 q^{-2} \\ -0.075 q^{-72}+0.081 q^{-73} \\ -0.002 q^{-19}-0.016 q^{-20} \\ -0.011 q^{-38}-0.004 q^{-39}\end{array}\right]$.

If $y(t)$ is the water level $y_{n j}(19, t)$, then the parameters are

$A\left(19, q^{-1}\right)=1-1.015 q^{-1}+0.028 q^{-2}$,

$B\left(19, q^{-1}\right)=0.327 q^{-1}-0.031 q^{-2}$,

$P\left(19, q^{-1}\right)=\left[\begin{array}{c}-0.006 q^{-1}+0.009 q^{-2} \\ 0.049 q^{-98}-0.046 q^{-99} \\ 0.113 q^{-35}-0.121 q^{-36} \\ 0.170 q^{-8}-0.170 q^{-9}\end{array}\right]$.

If $y(t)$ is the water level $y_{n j}(20, t)$, then the parameters are

$A\left(20, q^{-1}\right)=1-1.661 q^{-1}+0.668 q^{-2}$,

$B\left(20, q^{-1}\right)=0.260 q^{-1}-0.163 q^{-2}$,

$P\left(20, q^{-1}\right)=\left[\begin{array}{c}-0.054 q^{-1}+0.059 q^{-2} \\ -0.068 q^{-95}+0.072 q^{-96} \\ 0.016 q^{-32}-0.028 q^{-33} \\ 0.020 q^{-14}-0.029 q^{-15}\end{array}\right]$.

It is noticed that the control input $u(i)$ is zero during most of the experimental period, which may result in difficulty in identifying $A\left(q^{-1}\right)$. In ARX model identification, the data should satisfy the persistence of exciting (Ljung, 1999). The data for model identification and validation are selected from the real time operational data. In the paper only the data that vary with respect to time are selected to avoid unexciting. Here carefully selected data are assumed to be persistently exciting without proof and the parameters $A\left(q^{-1}\right), B\left(q^{-1}\right)$ and $P\left(q^{-1}\right)$ are identified with the selected data.

When the identified model is used for 24-step ( $2 \mathrm{~h}$ ) ahead prediction, the result is shown in Figs. 3-6.

\subsection{Analysis}

From Table 1 and Fig. 2 it can be seen that the length of time delay is consistent with the distance between the measurement points. The longer the distance is, the longer the time delay is. This is because in the studied Enclosing Dike, the connection types of the measurement points (excluding the water levels outside the Enclosing Dike) are non-channel connections, whose time delay is influenced by hydraulic conductivity and distance. In the regional area of the Enclosing Dike, hydraulic conductivities are in a very narrow range for the materials are almost the same. So the time delays mainly reflect the difference in the distances. The identification result confirms this phenomenon.

Another phenomenon is that when a gate is open, the water level inside the gate varies with the change of outside the gate. It can be seen from Fig. 7 that the water levels inside and outside the gate are almost equal when the gate is open. This is easy to be understood. When there is a difference between the water levels, flow will occur to compensate for the difference. In the model, the flow rate through the gate is very small when the gate is open if it is calculated through the difference of the water levels at a specific time. Actually, the flow rate refers to the flow during a sampling

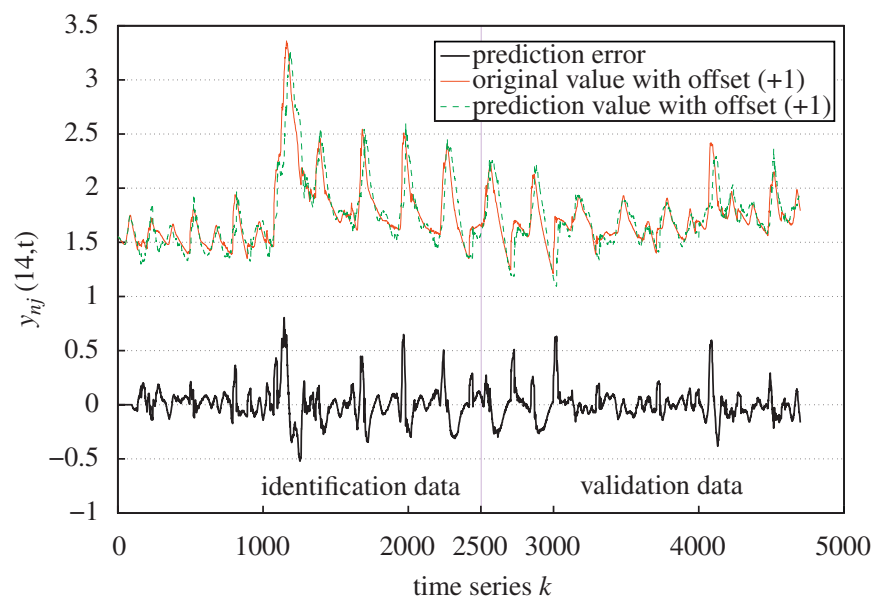

Fig. 3. Validation result for water level $y_{n j}(14, t)$.

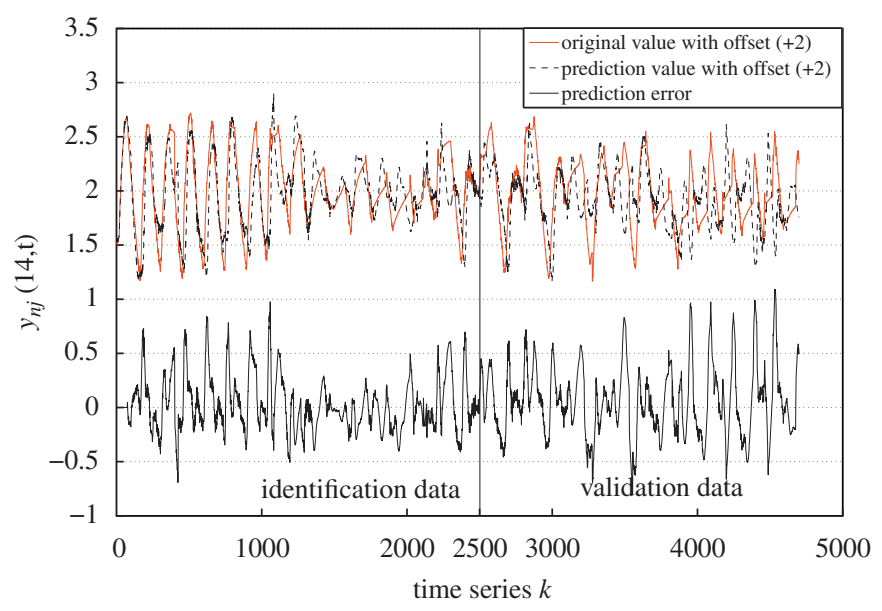

Fig. 4. Validation result for water level $y_{n j}(16, t)$.

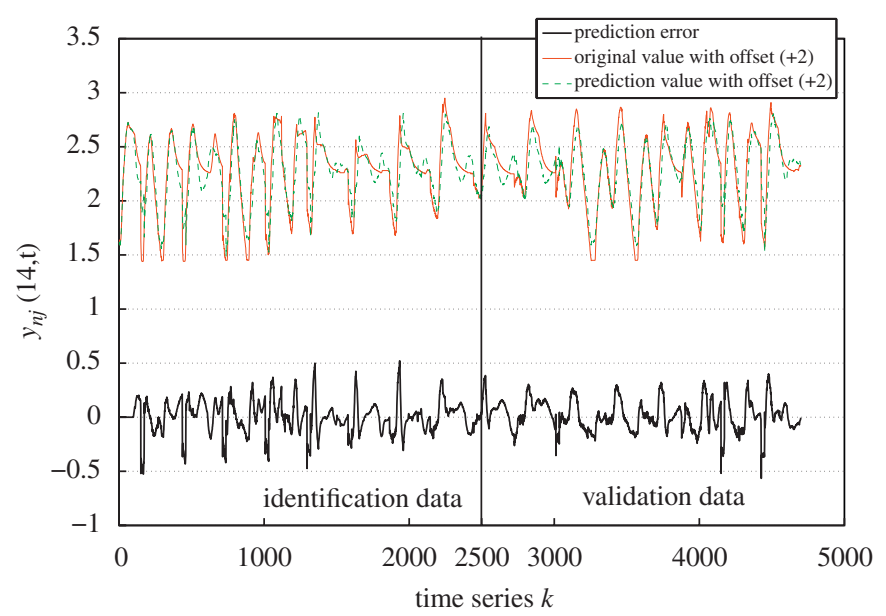

Fig. 5. Validation result for water level $y_{n j}(19, t)$.

period, so in the model, such a flow is calculated based on the difference between inside water level at present and outside water level at the next sampling time. Taking into consideration the offsets of the measurements, it is calculated based on the difference between the inside (or outside) water levels at present and at the next sampling time. The contribution to the inside 


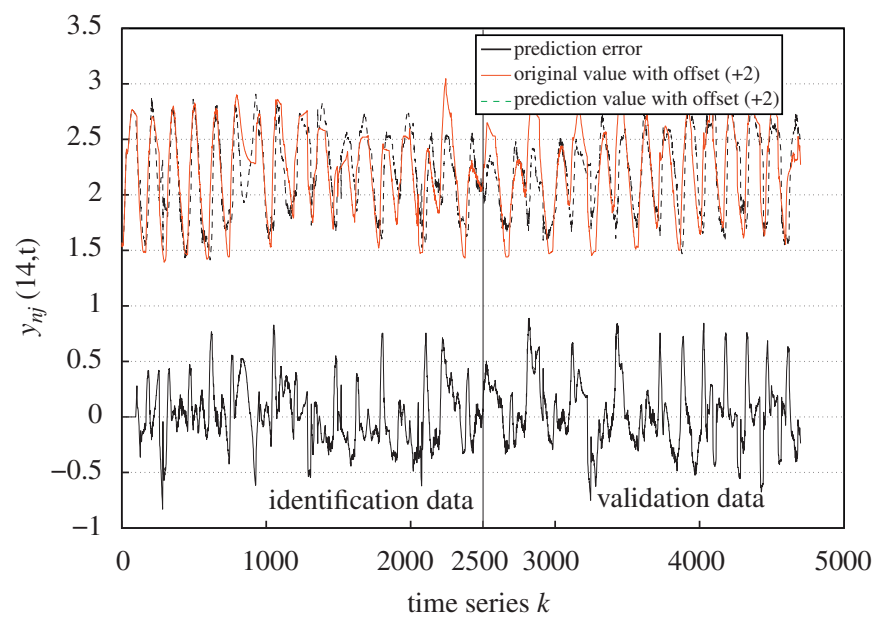

Fig. 6. Validation result for water level $y_{n j}(20, t)$.

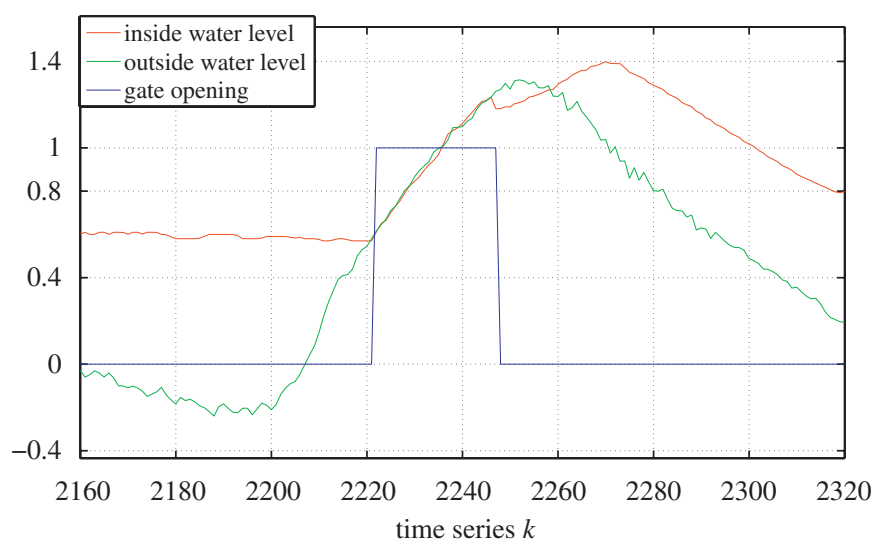

Fig. 7. Variation in water levels with gate opening.

water level variation is from the outside water level when the gate is open. Such a phenomenon is reflected on the parameter value of the flow rate through the gate, which is usually larger than other parameters in the model, as can be seen from Eqs. (11)-(14).

For a more accurate model, it can simply be assumed that when the gate is open, the inside water level is equal to the outside water level and is not related to other variables. When the gate is closed, the relationships with other variables are identified with experimental data.

When a gate is closed, the inside water level is influenced greatly by disturbances or unmodelled variables, for example, unmodelled water resources, rainfall, irrigation demands or evaporation. The fast variation of unmodelled variables leads to a large error in prediction. So, in further study, more variables, such as rainfall, the evaporation rate, water levels in other points of the area and irrigation flows, could be included in the model.

The statistics of prediction errors are shown in Table 2 for the identification period and Table 3 for the prediction period.

In comparing Table 2 with Table 3 , it can be seen that the prediction errors are not much worse in the prediction period than in the identification period, which means that the prediction errors mainly result from the unmodelled variables. With more variables included in the model, the errors will decrease. This proves the feasibility of the modelling methodology.

From Table 3, it is also seen that the errors in $2 \mathrm{~h}$-ahead prediction are acceptable in decision-making on the management and operation of the Enclosing Dike. When a particular security redundancy of the water level is considered, the operation can be
Table 2

Prediction error in identification period.

\begin{tabular}{lllll}
\hline & Min & Max & Mean & Std \\
\hline$y_{n j}(14, t)$ & -0.5216 & 0.8047 & 0.0130 & 0.1637 \\
$y_{n j}(16, t)$ & -0.2301 & 2.4509 & 0.7914 & 0.4076 \\
$y_{n j}(19, t)$ & -0.2701 & 2.4171 & 0.5088 & 0.3943 \\
$y_{n j}(20, t)$ & -0.4527 & 2.5366 & 0.5730 & 0.4583 \\
\hline
\end{tabular}

Table 3

Prediction error in prediction period.

\begin{tabular}{lrrrr}
\hline & Min & Max & \multicolumn{1}{l}{ Mean } & \multicolumn{1}{l}{ Std } \\
\hline$y_{n j}(14, t)$ & -0.3832 & 0.6324 & -0.0035 & 0.1366 \\
$y_{n j}(16, t)$ & 0.0489 & 1.8466 & 0.7894 & 0.3014 \\
$y_{n j}(19, t)$ & -0.1309 & 1.0925 & 0.4181 & 0.2422 \\
$y_{n j}(20, t)$ & -0.2582 & 1.2037 & 0.5135 & 0.3143 \\
\hline
\end{tabular}

planned based on the model (10) and the prediction error will be tolerant. With a decrease in the prediction period and an increase in measurement points, accuracy will be improved.

\subsection{Future work}

Further study based on a specific dam-river network system will proceed with some application issues being considered. The other direction is the application of a model based on the proposed modelling methodology in the management and operation from the point of view of the whole network system.

Model (9) facilitates the study on the control problem of damriver network systems. With this model, different kinds of control problem can be formulated for a dam-river network system. For example, a local output feedback for water level regulation can easily be formulated in the following ways: Local water lever regulation: To find a controller in the following form:

$u(t)=\mathscr{L}(y(t), w(t))$,

such that the water level $\lim _{t \rightarrow \infty}\left(y(t)-y_{c}(t)\right)=0$, where $y_{c}(t)$ is the reference output.

For local water level safety control, the objective is to find a controller in the form (15) such that the output $y(t)$ is kept in the safety range $[\bar{Y}, Y]$.

When several points (indexes are $1, \ldots, N$ ) are considered in a study, there are coupled equations in the form of (9) composing a model for the network system. Then, based on the series of models, the control problem can be formulated from a global viewpoint. For example, the optimal water level regulation can be formulated as follows. of

Optimal water level regulation: To find a controller in the form

$u(t)=\mathscr{L}(y(t), w(t))$,

such that the performance function

$f=\int\left(y^{\mathrm{T}} \Lambda y+u^{\mathrm{T}} \Pi u\right) \mathrm{d} t$,

is minimized with $\Lambda, \Pi$ as weighting matrices.

Of course, many other formulations can be proposed based on the mathematical model (9).

These control problems will be dealt with elsewhere. 


\section{Conclusions}

A very simple modelling methodology is proposed in this paper for modelling natural dam-river network systems. In the model, only water level measurements are used in combination with some control variables, such as gate openings and pump flow rates.

Considering the complexity of a natural dam-river network system, this modelling methodology is very simple. The proposed model structure is based on some hydraulic principles. With the experimental data, the parameters in the model can be identified.

An application example is given to show the feasibility of the modelling methodology. Some application issues in the example are considered, such as data synchronization, data offsets and missing data. Preprocess of the data is necessary.

With the analysis of the model identification and validation results, the physical significance of time delays is discussed together with the hydraulic characteristics. Some improvement measures for the model are proposed, based on the analysis results.

The accuracy of such a model in the application of prediction is discussed. The model shown in this paper is good enough for $2 \mathrm{~h}-$ ahead prediction. This means that decisions on management and operation can be made based on $2 \mathrm{~h}$-ahead prediction. Compared with the present decision-making mechanism (based on present states), it is an improvement. Moreover, the existing decisionmaking is based on present local measurements, while with the proposed model, such decision-making can be based on the measurements in the network system. Optimal operation is possible from the point of view of the network system.

\section{Acknowledgements}

This material is based upon work supported by the National Research Foundation of South Africa within the framework of the joint China/South Africa Science and Technology Agreement under Grant number 2075481.

The authors would like to thank Mr. Z. Wang at Wuhan University for his kindness in providing the data, as well as Prof. Y. $\mathrm{Xu}$ at the University of the Western Cape for the useful comments.

\section{References}

Balogun, O. S., Hubbard, M., \& De Vries, J. J. (1988). Automatic control flow using linear quadratic regulator theory. Journal of Hydraulic Engineering, 114(1), 75-102.

Baume, J.-P., \& Sau, J. (1997). Study of irrigation canal dynamics for control purposes. In International workshop on the regulation of irrigation canals: State of the art of research and application (pp. 3-12).

Chentouf, B., Xu, C.-Z., \& Boulbrachene, M. (2001). Robust regulation of a river reach governed by Hayami model. In Proceedings of the 40th IEEE conference on decision and control (Vol. 5, pp. 4962-4967).

Chow, V. (1959). Open-channel hydraulics. New York: McGraw-Hill.

Corriga, G., Patta, F., Sanna, S., \& Usai, G. (1979). A mathematical model for openchannel networks. Applied Mathematical Modelling, 3(1), 51-54.

Corriga, G., Sanna, S., \& Usai, G. (1983). Sub-optimal constant-volume control for open channel networks. Applied Mathematical Modelling, 7(4), 262-267.

Duviella, E., Charbonnaud, P., Chiron, P., \& Carrillo, F. J. (2005). Supervised internal multi-model control of a dam-gallery open-channel system. In European control conference. CDC-ECC '05. 44th IEEE conference on decision and control (pp. 1887-1892)
Elfawal-Mansour, H., Georges, D., \& Ohnishi, N. (2000). Optimal control of an open channel irrigation system based on nonlinear models. In Proceedings of TENCON 2000 (Vol. 3, pp. 308-313).

Eurén, K., \& Weyer, E. (2007). System identification of open water channels with undershot and overshot gates. Control Engineering Practice, 15(7), 813-824.

Garcia, A., Hubbard, M., \& De Vries, J. J. (1992). Open channel transient flow control by discrete time LQR methods. Automatica, 28(2), 255-264.

Georges, D. (1994). Nonlinear model identification and state-observer design for water distribution systems. In Proceedings of international conference on control (Vol. 2, pp. 887-894).

Li, Y., Cantoni, M., \& Weyer, E. (2005). On water-Level error propagation in controlled irrigation channels. In 44th IEEE decision and control, 2005 and 2005 European control conference. CDC-ECC '05 (pp. 2101-2106).

Litrico, X. (2001). Robust flow control of single input multiple-outputs regulated rivers. Journal of Irrigation and Drainage Engineering, 127(5), 281-286.

Litrico, X., \& Georges, D. (1997). Nonlinear identification of an irrigation system. In Proceeding of the 36th IEEE conference on decision and control (pp. 852-857).

Litrico, X., \& Georges, D. (1999a). Robust continuous-time and discrete-time flow control of a dam-river system. (I) Modelling. Applied Mathematical Modelling, 23(11), 809-827.

Litrico, X., \& Georges, D. (1999b). Robust continuous-time and discrete-time flow control of a dam-river system. (II) Controller design. Applied Mathematical Modelling, 23(11), 829-846.

Litrico, X., \& Georges, D. (2001). Robust LQG control of single input multipleoutputs dam-river systems. International Journal of System Science, 32(6), 795-805.

Litrico, X., Georges, D., \& Trouvat, J.-L. (1998). Modelling and robust control of a dam-river system. In IEEE international conference on systems, man, and cybernetics (Vol. 4, pp. 3862-3867).

Ljung, L. (1999). System identification: Theory for the user. NJ: Prentice-Hall.

Mareels, I., Weyer, E., Ooi, S. K., Cantoni, M., Li, Y., \& Nair, G. (2005). Systems engineering for irrigation systems: Successes and challenges. Annual Reviews in Control, 29(2), 191-204.

Maxwell, M., \& Warnick, S. (2006). Modeling and identification of the Sevier River System. In American control conference (pp. 5342-5347).

Ooi, S. K., Krutzen, M. P. M., \& Weyer, E. (2005). On physical and data driven modelling of irrigation channels. Control Engineering Practice, 13(4), 461-471.

Ooi, S. K., \& Weyer, E. (2001). Closed loop identification of an irrigation channel. In Proceedings of the 40th IEEE conference on decision and control (Vol. 5, pp. 4338-4343)

Ooi, S. K., Weyer, E., \& Campi, M. C. (2003). Finite sample quality assessment of system identification models of irrigation channels. In Proceedings of 2003 IEEE conference on control applications (Vol. 2, pp. 1239-1244).

Paravan, D., Stokelj, T., \& Golob, R. (2004). Improvements to the water management of a run-of-river HPP reservoir: Methodology and case study. Control Engineering Practice, 12(4), 377-385.

Sawadogo, S., Faye, R.M., Benhammou, A., \& Akouz, K. (2000). Decentralized adaptive predictive control of multi-reach irrigation canal. In IEEE international conference on systems, man and cybernetics (Vol. 5, pp. 3437-3442).

Sawadogo, S., Faye, R.M., Malaterre, P.-O., \& Mora-Camino, F. (1998). Decentralized predictive controller for delivery canals. In IEEE international conference on systems, man and cybernetics (Vol. 4, pp. 3880-3884).

Schuurmans, J., Bosgra, O. H., \& Brouwer, R. (1995). Open-channel flow model approximation for controller design. Applied Mathematical Modelling, 19(9), 525-530.

Schuurmans, J., Hof, A., Dijkstra, S., Bosgra, O. H., \& Brouwer, R. (1999). Simple water level controller for irrigation and drainage canals. Journal of Irrigation and Drainage Engineering, 125(4), 189-195.

Todd, D. K., \& Mays, L. W. (1999). Groundwater hydrology. NJ: Wiley.

Toudeft, A., \& Gallinari, P. (1996). Control of a nonminimum phase system with varying time-delay using neural networks. In Proceedings of the 1996 IEEE international symposium on intelligent control (pp. 295-300).

Toudeft, A., \& Gallinari, P. (1997). Neural and adaptive controllers for a nonminimum phase varying time-delay system. Artificial Intelligence in Engineering, 11(4), 431-439.

Weyer, E. (2001). System identification of an open water channel Control Engineering Practice, 9(12), 1289-1299.

Weyer, E. (2003a). Controller configurations for irrigation channels derived from sequential minimum variance control. In Proceedings of IEEE conference on control applications (Vol. 2, pp. 1209-1214).

Weyer, E. (2003b). LQ control of an irrigation channel. In Proceedings of 42nd IEEE conference on decision and control (Vol. 1, pp. 750-755).

Zhuan, X., \& Xia, X. (2007). Models and control methodologies of open water flow dynamics: A survey. In Proceedings of IEEE Africon 2007. 